\title{
Interferência e controle de milho voluntário tolerante ao glifosato na cultura da soja
}

\author{
Ramiro Fernando López-Ovejero( ${ }^{(1)}$, Daniel Jorge Soares(1), Natália Cipolini Oliveira(1), Ibene Takao Kawaguchi( ${ }^{(1)}$, \\ Geraldo Ubirajara Berger ${ }^{(1)}$, Saul Jorge Pinto de Carvalho( ${ }^{(2)}$ e Pedro Jacob Christoffoleti ${ }^{(3)}$ \\ (1)Monsanto do Brasil, Avenida Nações Unidas, oo 12.901, Torre Norte, CEP 04578-910 São Paulo, SP, Brasil. E-mail: \\ ramiro.f.ovejero@monsanto.com, daniel.j.soares@monsanto.com, natalia.oliveira@monsanto.com, ibene.t.kawaguchi@monsanto.com, \\ geraldo.u.berger@monsanto.com ${ }^{(2)}$ Instituto Federal do Sul de Minas Gerais, Campus Machado, Rodovia Machado-Paraguaçu, Km 3, \\ CEP 37750-000 Machado, MG, Brasil. E-mail: sjpcarvalho@yahoo.com.br ${ }^{(3)}$ Universidade de São Paulo, Escola Superior de Agricultura Luiz \\ de Queiroz, Avenida Pádua Dias, no 11, Caixa Postal 9, CEP 13418-900 Piracicaba, SP, Brasil. E-mail: pjchrist@usp.br
}

Resumo - O objetivo deste trabalho foi estimar o efeito da infestação de milho voluntário tolerante ao glifosato na produtividade da cultura da soja, bem como avaliar o controle dessas plantas com o herbicida cletodim. Seis experimentos foram desenvolvidos, na safra de 2013/2014, nos estados da Bahia, de Minas Gerais, de Mato Grosso, do Paraná, do Rio Grande do Sul e de São Paulo. Espigas de milho sem palha, segmentadas ao meio, foram uniformemente distribuídas nas parcelas, à densidade de 1, 2 ou 4 segmentos por metro quadrado. Como tratamento herbicida, utilizou-se cletodim, nas seguintes doses: aplicação única, $108 \mathrm{~g} \mathrm{ha}^{-1}$, sobre a soja com três trifólios (3T); aplicação única, $108 \mathrm{~g} \mathrm{ha}^{-1}$, sobre soja com seis trifólios (6T); e duas aplicações, 108 e $84 \mathrm{~g} \mathrm{ha}^{-1}$, sobre soja nos estágios 3T e 6T. Adicionou-se, ainda, um tratamento com a soja plenamente capinada. $\mathrm{O}$ aumento de densidade dos segmentos de espiga reduziu a produtividade da soja em até $69,9 \%$. A produtividade da soja foi assegurada com o controle eficaz das plantas voluntárias de milho tolerante ao glifosato, oriundas de espigas, em aplicação única de cletodim $\left(108 \mathrm{~g} \mathrm{ha}^{-1}\right)$ no estádio $6 \mathrm{~T}$; ou por meio de duas aplicações sequenciais do graminicida, com a soja em 3T e 6T.

Termos para indexação: Glycine max, Zea mays, cletodim, competição, graminicida.

\section{Interference and control of glyphosate-tolerant volunteer corn in soybean crop}

\begin{abstract}
The objective of this work was to estimate the infestation effect of glyphosate-tolerant volunteer corn in soybean yield, as well as to evaluate the control of these plants with the herbicide clethodim. Six experiments were carried out in the 2013/2014 season, in the following Brazilian states: Bahia, Minas Gerais, Mato Grosso, Paraná, Rio Grande do Sul, and São Paulo. Corn ears without straw, cut in half, were uniformly distributed in the plots at the density of 1,2 , or 4 segments per square meter. As herbicide treatment, clethodim was applied at the following doses: a single application, at $108 \mathrm{~g} \mathrm{ha}^{-1}$, on soybean with three trifoliate leaves (3T); a single application, at $108 \mathrm{~g} \mathrm{ha}^{-1}$, on soybean with six trifoliate leaves (6T); and two applications, at 108 and $84 \mathrm{~g} \mathrm{ha}^{-1}$, on soybean at the $3 \mathrm{~T}$ and $6 \mathrm{~T}$ stages. A handweeded soybean treatment was also included. The increased density of corn ear segments reduced soybean yield up to $69.9 \%$. Soybean yield was warranted by the efficient control of glyphosate-tolerant volunteer corn, originated from ears, with a single clethodim application at $108 \mathrm{~g} \mathrm{ha}^{-1}$ on soybean at the 6T stage, or using two sequential applications of this graminicide on soybean at $3 \mathrm{~T}$ and $6 \mathrm{~T}$.
\end{abstract}

Index terms: Glycine max, Zea mays, clethodim, competition, graminicide.

\section{Introdução}

$\mathrm{Na}$ agricultura brasileira atual, tem-se observado a intensificação dos sistemas produtivos, com o objetivo de alcançar maior rendimento agrícola e lucratividade financeira. Para tanto, destaca-se a repetida sucessão soja (Glycinemax) - milho (Zea mays), nas consecutivas safras, com destaque para regiões como o oeste do Paraná, e os estados de Mato Grosso, Mato Grosso do
Sul e Goiás (Petter et al., 2015). A semeadura do milho como segunda safra, após a colheita da soja, também conhecido como milho "safrinha", é uma realidade no Brasil e ocupa áreas expressivas de produção (Artuzi \& Contiero, 2006; Petter et al., 2015).

Nesses sistemas produtivos, os restos de grãos e de segmentos de espigas com grãos são comuns, durante o processo de colheita do milho, e dão origem a plantas voluntárias na cultura subsequente, também conhecida 
como plantas guaxas ou tigueras (Costa et al., 2014; Marca et al., 2015; Petter et al., 2015). Os grãos soltos ou espigas perdidas na colheita de milho safrinha podem permanecer no solo, com viabilidade de germinação e emergência, durante toda a estação seca (período invernal). Após o início da estação chuvosa e a semeadura da cultura subsequente, ou seja, primeira cultura da safra seguinte, esses grãos resultam no estabelecimento de plantas voluntárias, o que causa significativa interferência negativa na produtividade da cultura, tal como as plantas daninhas, em razão da competição por recursos para o crescimento, como água, luz e nutrientes. Beckett \& Stoller (1988) destacam que, em áreas de plantio direto, este problema é ainda mais importante, pois não há revolvimento do solo, de modo que os grãos são mantidos sobre a superfície da área, aptos à germinar e se estabelecer como plantas voluntárias.

Deen et al. (2006) comentam que o milho voluntário é um problema potencial sempre que for sucedido pela cultura da soja. Como consequência, essa interferência pode provocar significativa redução de produtividade da cultura da soja (Young \& Hart, 1997). Na prática, se reconhece que a presença de segmentos de espigas, diferentemente da presença de grãos individualizados, dificulta o manejo, pois, quando no solo, as sementes nas espigas não debulhadas apresentam desuniformidade de emergência, o que resulta em sucessivos fluxos. Deen et al. (2006) ponderam que o milho voluntário oriundo de grãos ainda ligados às espigas predomina em áreas em que ocorreu acamamento da colheita e, nesta situação, o milho voluntário ocorre em "touceiras" que, na prática, proporcionam alta densidade de plantas no local em que se encontra o segmento da espiga. Embora a ocorrência de milho voluntário oriundo de espigas seja muito comum nas lavouras de soja, são escassos os trabalhos que abordam esta importante condição de cultivo.

A convivência de plantas voluntárias com a cultura em sucessão pode resultar em perdas diretas ou indiretas, inclusive dificuldade de colheita, competição por recursos de crescimento e alternativa para hospedagem de insetos e patógenos (Beckett \& Stoller, 1988; York et al., 2004; Deen et al., 2006). A ocorrência de milho voluntário, na cultura da soja, foi documentada muito antes do surgimento dos eventos de transgenia que conferem às culturas tolerância a herbicidas (Andersen, 1976; Beckett \& Stoller, 1988). No entanto, as formas de manejo tiveram de ser modificadas com a liberação de híbridos de milho tolerantes ao glifosato, principalmente, quando a cultura em sucessão também é tolerante ao produto. Assim, atualmente, é comum a infestação de milho voluntário tolerante a herbicidas, nas culturas semeadas em sucessão ou rotação também tolerantes aos mesmos herbicidas (Thomas et al., 2007; Clewis et al., 2008; Steckel et al., 2009; Petter et al., 2015).

O glifosato é uma alternativa eficaz para o controle de milho voluntário, em lavouras de soja tolerante a este herbicida (soja RR). Porém, com o advento do milho também tolerante a glifosato, essa alternativa foi inviabilizada, o que exige a aplicação de herbicidas de outros mecanismos de ação, com destaque para os graminicidas (Beckett \& Stoller, 1988; Young \& Hart, 1997; Deen et al., 2006; Soltani et al., 2006; Marca et al., 2015). A adoção de graminicidas também é uma alternativa para o controle de biótipos de plantas daninhas resistentes ao glifosato, com destaque para o azevém (Lolium multiflorum) e o capim-amargoso (Digitaria insularis) (Maciel et al., 2013; Costa et al., 2014).

O objetivo deste trabalho foi estimar o efeito da infestação de milho voluntário tolerante ao glifosato na produtividade da cultura da soja, bem como avaliar o controle destas plantas com o herbicida cletodim.

\section{Material e Métodos}

Seis experimentos semelhantes foram desenvolvidos com a cultura da soja, na safra de 2013/2014, cada um deles realizado em um dos seguintes municípios brasileiros: Luís Eduardo Magalhães, BA (LEM); Cachoeira Dourada, MG (CCH); Sorriso, MT (SOR); Rolândia, PR (ROL); Não-Me-Toque, RS (NMT); e Santa Cruz das Palmeiras, SP (SCP). Em LEM, utilizou-se a cultivar de soja 'M8527RR', semeada em 13/12/2013; em CCH, adotou-se a cultivar 'M76399RR', semeada em 7/11/2013; em SOR, a cultivar 'AS 3820IPRO' foi semeada em 14/11/2013; em ROL, utilizou-se a cultivar 'M6210IPRO', semeada em 05/12/2013; em NMT, adotou-se a cultivar 'BMX ATIVA RR', semeada em 14/11/2013; e, em SCP, a cultivar 'M7908RR' foi semeada em 24/10/2013.

Em todas as localidades, a cultura da soja foi semeada em espaçamento de 0,50 m entre linhas. Todas as áreas foram devidamente fertilizadas e mantidas em perfeitas condições fitossanitárias. Em todos os experimentos, 
utilizou-se o delineamento experimental de blocos ao acaso, com treze tratamentos e quatro repetições. As parcelas experimentais consistiram de quatro linhas de soja, com $2 \mathrm{~m}$ de comprimento. As duas linhas centrais das parcelas foram consideradas como área útil, no total de $2 \mathrm{~m}^{2}$.

Os tratamentos experimentais consistiram de combinações entre a presença de plantas voluntárias de milho tolerante ao herbicida glifosato (híbrido DKB 390YG RR2) e medidas de manejo químico destas plantas (Tabela 1). Para simular a interferência de plantas voluntárias de milho nas lavouras de soja, espigas sem palha foram quebradas ao meio (transversal) e distribuídas uniformemente nas parcelas experimentais. Essas espigas foram plantadas em condição semi-incorporada $(50 \%)$, à densidade de 1, 2 ou 4 segmentos $\mathrm{m}^{-2}$, no mesmo dia da semeadura da cultura. Em complementação, adicionou-se um tratamento em que a soja foi mantida plenamente capinada, sem a presença de milho voluntário e plantas daninhas (testemunha capinada).

Como alternativa de controle do milho, adotaram-se pulverizações de cletodim. Realizaram-se as seguintes aplicações de cletodim: única, a $108 \mathrm{~g} \mathrm{ha}^{-1}$, sobre soja em estádio de três trifólios (3T); única, a $108 \mathrm{~g} \mathrm{ha}^{-1}$, sobre soja com seis trifólios (6T); e duas aplicações, a 108 e $84 \mathrm{~g} \mathrm{ha}^{-1}$, sobre soja em 3T e 6T. No momento

Tabela 1. Tratamentos experimentais, instalados nos estados da Bahia, Minas Gerais, Mato Grosso, Paraná, Rio Grande do Sul e São Paulo.

\begin{tabular}{lccc}
\hline Tratamento & $\begin{array}{c}\text { Segmentos de } \\
\text { espigas por } \mathrm{m}^{2(1)}\end{array}$ & $\begin{array}{c}\text { Herbicida cletodim } \\
\left(\mathrm{g} \mathrm{ha}^{-1}\right)\end{array}$ & $\begin{array}{c}\text { Momento de } \\
\text { aplicação }\end{array}$ \\
\hline 1 & Testemunha absoluta capinada & - \\
2 & 1 & Ausente & - \\
3 & 2 & Ausente & - \\
4 & 4 & Ausente & - \\
5 & 1 & 108 & $3 \mathrm{~T}^{(3)}$ \\
6 & 2 & 108 & $3 \mathrm{~T}$ \\
7 & 4 & 108 & $3 \mathrm{~T}$ \\
8 & 1 & 108 & $6 \mathrm{~T}^{(4)}$ \\
9 & 2 & 108 & $6 \mathrm{~T}$ \\
10 & 4 & 108 & $6 \mathrm{~T}$ \\
11 & 1 & 108 e 84 & $3 \mathrm{~T} \mathrm{e} 6 \mathrm{~T}$ \\
12 & 2 & 108 e 84 & $3 \mathrm{~T} \mathrm{e} \mathrm{6T}$ \\
13 & 4 & 108 e 84 & $3 \mathrm{~T} \mathrm{e} 6 \mathrm{~T}$ \\
\hline
\end{tabular}

${ }^{(1)}$ Segmentos referentes às espigas despalhadas e quebradas ao meio. ${ }^{(2)}$ Testemunha sem espigas, sem aplicação de herbicidas, plenamente capinada. ${ }^{(3)} 3 \mathrm{~T}$, soja com três trifólios verdadeiros. ${ }^{(4)} 6 \mathrm{~T}$, soja com seis trifólios verdadeiros. da primeira aplicação de cletodim, as plantas de milho oriundas de espigas estavam com $15 \mathrm{~cm}$ de altura e 1 a 2 folhas verdadeiras. Na segunda aplicação, identificaram-se plantas de milho com 20 a $25 \mathrm{~cm}$ e 3 a 4 folhas verdadeiras (Tabela 1).

A aplicação dos tratamentos foi realizada com auxílio de um pulverizador costal pressurizado com $\mathrm{CO}_{2}$, acoplado à barra de aplicação com quatro pontas do tipo TT110.015, espaçadas de $0,50 \mathrm{~m}$, reguladas para volume de calda proporcional a $120 \mathrm{~L} \mathrm{ha}^{-1}$. Adicionouse adjuvante à base de óleo mineral a $0,5 \% \mathrm{v} / \mathrm{v}$ à calda de todos os tratamentos herbicidas. Todas as parcelas de soja foram mantidas livres da competição com plantas daninhas, com exceção das plantas de milho voluntário tolerante a glifosato, por meio de duas aplicações de glifosato a $720 \mathrm{~g} \mathrm{ha}^{-1}$, realizadas aos 14 e 28 dias após a semeadura da soja.

As variáveis analisadas foram: controle percentual das plantas de milho, aos 28 dias após a segunda aplicação (DAA) de cletodim; controle das plantas de milho em pré-colheita da soja; estande (plantas $\mathrm{m}^{-2}$ ) da soja; e produtividade $\left(\mathrm{kg} \mathrm{ha}^{-1}\right)$ da soja. Para as avaliações de controle percentual, utilizou-se uma escala de notas variável entre 0 (ausente) e 100\% (plantas mortas). Na maturação fenológica da soja, realizou-se a contagem do estande da cultura nas duas linhas centrais, bem como a colheita da área útil das parcelas. A produtividade da cultura da soja foi obtida padronizando-se a umidade dos grãos para 13\%.

Todos os dados foram submetidos à aplicação do teste $\mathrm{F}$, para a análise da variância, a $5 \%$ de probabilidade. Em seguida, quando detectada a significância do efeito de tratamentos, os dados foram submetidos ao teste de agrupamento de médias de Scott-Knott, também a 5\% de probabilidade (Scott \& Knott, 1974). Realizou-se a avaliação da viabilidade da análise dos dados por grupo de experimentos, por meio do teste de homogeneidade de variâncias de Hartley (Pearson \& Hartley, 1956).

\section{Resultados e Discussão}

Inicialmente, aplicou-se o teste de homogeneidade de variâncias de Hartley (Pearson \& Hartley, 1956) sobre os dados experimentais, para a análise por grupo de experimentos. Contudo, foi detectada a heterogeneidade de variâncias entre as áreas experimentais, o que exigiu que estas fossem analisadas separadamente.

Nas Tabelas 2 e 3, estão apresentados os valores de controle obtidos sobre o milho voluntário após a 
aplicação de cletodim, na avaliação aos 28 dias após a segunda aplicação (Tabela 2) ou em pré-colheita da soja (Tabela 3). Nota-se que o padrão de controle registrado é bastante semelhante nas duas avaliações. Observase que os menores níveis de controle foram obtidos quando se realizou apenas uma aplicação de cletodim, com a cultura da soja em 3T. Nesta condição, os níveis de controle mais baixos - inferiores a $20 \%$ - foram observados na localidade de MG (Tabelas 2 e 3). Estes resultados podem ser consequência de um período de seca, registrado na região após a semeadura da soja, que retardou a germinação das plantas voluntárias de milho nas espigas, que tiveram maior germinação após a primeira aplicação do graminicida.

Ainda, considerando-se uma aplicação isolada do graminicida, os melhores níveis de controle do milho voluntário foram alcançados, em geral, quando a aplicação foi realizada em estádio 6T da soja, porém, variaram em razão do número de segmentos de espigas presentes nas áreas (Tabelas 2 e 3). Para as densidades de 1 ou 2 segmentos $\mathrm{m}^{-2}$, apenas uma aplicação de cletodim, com a soja em 6T, foi suficiente para o adequado controle do milho voluntário, com níveis de controle sempre superiores a $80 \%$, em cinco das seis localidades. Neste sentido, vale ressaltar que o herbicida cletodim não apresenta ação residual no solo (Rodrigues \& Almeida, 2011), de modo que novos fluxos de milho não são controlados após cada aplicação. Assim, considera-se que a aplicação do graminicida realizada em $6 \mathrm{~T}$ foi eficaz sobre o maior número de plantas de milho e garantiu menor competição geral na área.

Todavia, outro fator que pode ter contribuído para o melhor controle, quando as aplicações foram realizadas no $6 \mathrm{~T}$, foi o fechamento das entrelinhas da soja. Assim, após essa aplicação, novos fluxos de milho foram inibidos pelo efeito de sombreamento, o que não ocorreu quando as aplicações foram realizadas no 3T, em que os novos fluxos que ocorreram após a aplicação não foram controlados, e o sombreamento da cultura não produziu efeito. Para a densidade de 4 segmentos de espigas $\mathrm{m}^{-2}$, houve diferenciação de controle em três das seis localidades estudadas, o que indica a necessidade de maior intervenção química para a plena eliminação das plantas voluntárias (Tabelas 2 e 3).

Em São Paulo, o controle máximo obtido com uma aplicação de cletodim em 6T foi de $85 \%$ (Tabela 3). Este resultado pode estar relacionado ao maior índice de área foliar, observado visualmente na variedade de soja utilizada, que pode ter proporcionado o efeito "guarda-chuva", durante a aplicação do graminicida

Tabela 2. Controle percentual de milho voluntário, germinado a partir de segmentos de espigas distribuídas em três densidades, nas entrelinhas da cultura da soja, avaliado aos 28 dias após a segunda aplicação do herbicida cletodim, em seis estados brasileiros ${ }^{(1)}$.

\begin{tabular}{|c|c|c|c|c|c|c|c|c|}
\hline \multirow{2}{*}{$\begin{array}{l}\text { Segmentos de } \\
\text { espigas por m }{ }^{2(2)}\end{array}$} & \multirow{2}{*}{$\begin{array}{l}\text { Cletodim } \\
\left(\mathrm{g} \mathrm{ha}^{-1}\right)\end{array}$} & \multirow{2}{*}{$\begin{array}{l}\text { Momento de } \\
\text { aplicação }\end{array}$} & \multicolumn{6}{|c|}{ Local de desenvolvimento do experimento ${ }^{(3)}$} \\
\hline & & & BA & MG & MT & PR & $\mathrm{RS}$ & SP \\
\hline \multicolumn{3}{|c|}{ Testemunha absoluta capinada ${ }^{(4)}$} & $100,0 \mathrm{a}$ & $100,0 \mathrm{a}$ & $100,0 \mathrm{a}$ & $100,0 \mathrm{a}$ & $100,0 \mathrm{a}$ & $100,0 \mathrm{a}$ \\
\hline 1 & Ausente & - & $0,0 \mathrm{~d}$ & $0,0 \mathrm{~d}$ & $0,0 \mathrm{e}$ & $0,0 \mathrm{e}$ & $0,0 \mathrm{~d}$ & $0,0 \mathrm{~g}$ \\
\hline 2 & Ausente & - & $0,0 \mathrm{~d}$ & $0,0 \mathrm{~d}$ & $0,0 \mathrm{e}$ & $0,0 \mathrm{e}$ & $0,0 \mathrm{~d}$ & $0,0 \mathrm{~g}$ \\
\hline 4 & Ausente & - & $0,0 \mathrm{~d}$ & $0,0 \mathrm{~d}$ & $0,0 \mathrm{e}$ & $0,0 \mathrm{e}$ & $0,0 \mathrm{~d}$ & $0,0 \mathrm{~g}$ \\
\hline 1 & 108 & $3 \mathrm{~T}^{(5)}$ & $78,8 \mathrm{~b}$ & $18,9 b$ & $81,3 \mathrm{c}$ & $61,3 \mathrm{c}$ & $73,8 \mathrm{c}$ & $76,3 d$ \\
\hline 2 & 108 & $3 \mathrm{~T}$ & $72,5 \mathrm{c}$ & $11,3 \mathrm{c}$ & $77,5 \mathrm{~d}$ & $65,0 \mathrm{c}$ & $76,3 c$ & $61,3 \mathrm{f}$ \\
\hline 4 & 108 & $3 \mathrm{~T}$ & $71,3 \mathrm{c}$ & $10,0 \mathrm{c}$ & $76,3 \mathrm{~d}$ & $55,0 \mathrm{~d}$ & $75,0 \mathrm{c}$ & $57,5 \mathrm{f}$ \\
\hline 1 & 108 & $6 \mathrm{~T}^{(6)}$ & $95,3 \mathrm{a}$ & $98,0 \mathrm{a}$ & $99,5 \mathrm{a}$ & $98,3 \mathrm{a}$ & $85,0 \mathrm{~b}$ & $77,5 \mathrm{~d}$ \\
\hline 2 & 108 & $6 \mathrm{~T}$ & $93,8 \mathrm{a}$ & $98,0 \mathrm{a}$ & $99,5 \mathrm{a}$ & $97,5 \mathrm{a}$ & $85,0 \mathrm{~b}$ & $77,5 \mathrm{~d}$ \\
\hline 4 & 108 & $6 \mathrm{~T}$ & $95,8 \mathrm{a}$ & $98,0 \mathrm{a}$ & $92,5 b$ & $92,5 b$ & $85,0 \mathrm{~b}$ & $71,3 \mathrm{e}$ \\
\hline 1 & 108 e 84 & $3 \mathrm{~T}$ e $6 \mathrm{~T}$ & $95,8 \mathrm{a}$ & $98,0 \mathrm{a}$ & $98,5 \mathrm{a}$ & $100,0 \mathrm{a}$ & $97,3 \mathrm{a}$ & $86,3 \mathrm{c}$ \\
\hline 2 & 108 e 84 & $3 \mathrm{~T}$ e $6 \mathrm{~T}$ & $95,3 a$ & $98,0 \mathrm{a}$ & $99,5 \mathrm{a}$ & $100,0 \mathrm{a}$ & $98,0 \mathrm{a}$ & $92,5 b$ \\
\hline 4 & 108 e 84 & $3 \mathrm{~T}$ e $6 \mathrm{~T}$ & $95,0 \mathrm{a}$ & $98,0 \mathrm{a}$ & $93,3 b$ & $99,0 \mathrm{a}$ & $98,0 \mathrm{a}$ & $93,8 b$ \\
\hline \multicolumn{3}{|l|}{ Teste $\mathrm{F}_{\text {trat }}$} & $362,54 * *$ & $455,90 * *$ & $741,78 * *$ & $544,64 * *$ & $1.392,99 * *$ & $558,20 * *$ \\
\hline \multicolumn{3}{|c|}{ Coeficiente de variação (\%) } & 6,15 & 8,00 & 4,28 & 5,30 & 3,14 & 5,11 \\
\hline
\end{tabular}

${ }^{(1)}$ Médias seguidas por letras iguais, nas colunas, não diferem pelo teste de Scott-Knott, a 5\% de probabilidade. ${ }^{(2)}$ Segmentos referentes às espigas despalhadas e quebradas ao meio. ${ }^{(3)}$ Luís Eduardo Magalhães, BA; Cachoeira Dourada, MG; Sorriso, MT; Rolândia, PR; Não-Me-Toque, RS; Santa Cruz das Palmeiras, SP. ${ }^{(4)}$ Testemunha sem espigas, sem aplicação de herbicidas, plenamente capinada. ${ }^{(5)} 3 \mathrm{~T}$, soja com três trifólios verdadeiros. ${ }^{(6)} 6 \mathrm{~T}$, soja com seis trifólios verdadeiros. **Significativo a $1 \%$ de probabilidade pelo teste $\mathrm{F}$. 
sobre as plantas de milho, ou pode ser atribuído à ocorrência de maior número de fluxos de plantas de milho oriundas das espigas. Estes resultados justificam a importância de se evitarem as perdas de grãos e espigas de milho, antes e durante a colheita, para diminuir a ocorrência de espigas e grãos remanescentes nas áreas agrícolas. Puzzi (1986), por exemplo, relata que o atraso na época da colheita mecânica, após a maturação do milho, provoca a diminuição do teor de água dos grãos e, consequentemente, o aumento significativo das perdas.

Assim, considera-se que o manejo das plantas voluntárias de milho, na cultura da soja, começa ainda no período de condução da cultura do milho, com destaque para sua colheita. Segundo Bragachini \& Peiretti (2005), em estudo realizado na Argentina, a perda de milho observada na colheita pode alcançar valores de $65 \mathrm{~kg} \mathrm{ha}^{-1}$, o que equivale a $0,9 \%$ do total colhido. Em estudo desenvolvido no Brasil, a perda total ocorrida no processo de colheita mecanizada foi de até $9 \%$, que pode ter sido favorecida pelo atraso da colheita mecânica que, no caso do trabalho desenvolvido por Souza et al. (2006), foi de aproximadamente 30 dias após a maturação da cultura.

$\mathrm{Na}$ avaliação em pré-colheita da soja, para a maior densidade de plantas voluntárias, de 4 segmentos de espigas $\mathrm{m}^{-2}$, e em quatro das seis localidades, o melhor controle foi obtido, quando se realizaram duas aplicações de cletodim, com a soja em 3T e 6T (Tabela 3). Assim, resume-se o plano para o controle de milho voluntário, oriundo de espigas, em um padrão bastante claro e consistente: para a condição de baixa infestação, pode-se optar pela adoção de apenas uma aplicação de graminicida (cletodim), que deve ser realizada preferencialmente com a soja em estádio de 6T; para a condição de alta infestação, a maior probabilidade de sucesso será alcançada por meio de duas aplicações de cletodim, uma em $3 \mathrm{~T}$ e outra em 6T. Este padrão de controle para o milho voluntário oriundo de espiga é diferenciado em relação ao manejo de plantas germinadas de grãos soltos e justificado pelo maior número de fluxos de germinação dos grãos nas espigas.

Deen et al. (2006) também propuseram sistemas de manejo de milho voluntário, fundamentado na aplicação combinada de glifosato e graminicida. Neste caso, o herbicida quizalofope-P-etil foi o mais eficaz no controle de milho voluntário tolerante a glifosato, seguido por cletodim e fenoxaprope-P-etil. Relatam, ainda, que a aplicação conjunta de glifosato, graminicidas e adjuvantes não prejudicou a eficácia dos herbicidas ou a tolerância da soja. Soltani

Tabela 3. Controle percentual de milho voluntário, germinado a partir de segmentos de espigas ${ }^{(1)}$ distribuídas em três densidades, nas entrelinhas da cultura da soja, avaliado na pré-colheita da cultura da soja, em seis estados brasileiros ${ }^{(1)}$.

\begin{tabular}{|c|c|c|c|c|c|c|c|c|}
\hline \multirow{2}{*}{$\begin{array}{l}\text { Segmentos de } \\
\text { espigas por } \mathrm{m}^{2(2)}\end{array}$} & \multirow{2}{*}{$\begin{array}{l}\text { Cletodim } \\
\left(\mathrm{g} \mathrm{ha}^{-1}\right)\end{array}$} & \multirow{2}{*}{$\begin{array}{c}\text { Momento de } \\
\text { aplicação }\end{array}$} & \multicolumn{6}{|c|}{ Local de desenvolvimento do experimento $^{(3)}$} \\
\hline & & & BA & MG & MT & $\mathrm{PR}$ & RS & SP \\
\hline \multicolumn{2}{|c|}{ Testemunha absoluta capinada ${ }^{(4)}$} & & $100,0 \mathrm{a}$ & $100,0 \mathrm{a}$ & $100,0 \mathrm{a}$ & $100,0 \mathrm{a}$ & $100,0 \mathrm{a}$ & $100,0 \mathrm{a}$ \\
\hline 1 & Ausente & - & $0,0 \mathrm{c}$ & $0,0 \mathrm{~d}$ & $0,0 \mathrm{f}$ & $0,0 \mathrm{e}$ & $0,0 \mathrm{~d}$ & $0,0 \mathrm{~d}$ \\
\hline 2 & Ausente & - & $0,0 \mathrm{c}$ & $0,0 \mathrm{~d}$ & $0,0 \mathrm{f}$ & $0,0 \mathrm{e}$ & $0,0 \mathrm{~d}$ & $0,0 \mathrm{~d}$ \\
\hline 4 & Ausente & - & $0,0 \mathrm{c}$ & $0,0 \mathrm{~d}$ & $0,0 \mathrm{f}$ & $0,0 \mathrm{e}$ & $0,0 \mathrm{~d}$ & $0,0 \mathrm{~d}$ \\
\hline 1 & 108 & $3 \mathrm{~T}^{(5)}$ & $75,0 \mathrm{~b}$ & $8,8 b$ & $55,0 \mathrm{~d}$ & $55,0 \mathrm{c}$ & $51,3 \mathrm{c}$ & $76,3 b$ \\
\hline 2 & 108 & $3 \mathrm{~T}$ & $70,0 \mathrm{~b}$ & $6,3 \mathrm{c}$ & $45,0 \mathrm{e}$ & $50,0 \mathrm{c}$ & $60,0 \mathrm{~b}$ & $63,8 \mathrm{~b}$ \\
\hline 4 & 108 & $3 \mathrm{~T}$ & $72,5 b$ & $5,0 \mathrm{c}$ & $52,5 \mathrm{~d}$ & $42,5 \mathrm{~d}$ & $42,5 \mathrm{c}$ & $43,8 \mathrm{c}$ \\
\hline 1 & 108 & $6 \mathrm{~T}^{(6)}$ & $100,0 \mathrm{a}$ & $100,0 \mathrm{a}$ & $100,0 \mathrm{a}$ & $100,0 \mathrm{a}$ & $100,0 \mathrm{a}$ & $85,0 \mathrm{~b}$ \\
\hline 2 & 108 & $6 \mathrm{~T}$ & $99,0 \mathrm{a}$ & $100,0 \mathrm{a}$ & $100,0 \mathrm{a}$ & $100,0 \mathrm{a}$ & $100,0 \mathrm{a}$ & $76,3 b$ \\
\hline 4 & 108 & $6 \mathrm{~T}$ & $97,5 \mathrm{a}$ & $100,0 \mathrm{a}$ & $83,8 \mathrm{c}$ & $90,0 \mathrm{~b}$ & $76,3 b$ & $71,3 b$ \\
\hline 1 & 108 e 84 & $3 \mathrm{~T}$ e $6 \mathrm{~T}$ & $99,5 \mathrm{a}$ & $100,0 \mathrm{a}$ & $100,0 \mathrm{a}$ & $100,0 \mathrm{a}$ & $100,0 \mathrm{a}$ & $100,0 \mathrm{a}$ \\
\hline 2 & 108 e 84 & $3 \mathrm{~T}$ e $6 \mathrm{~T}$ & $98,8 \mathrm{a}$ & $100,0 \mathrm{a}$ & $100,0 \mathrm{a}$ & $100,0 \mathrm{a}$ & $100,0 \mathrm{a}$ & $100,0 \mathrm{a}$ \\
\hline$\underline{4}$ & 108 e 84 & $3 \mathrm{~T}$ e $6 \mathrm{~T}$ & $99,3 \mathrm{a}$ & $100,0 \mathrm{a}$ & $92,5 b$ & $100,0 \mathrm{a}$ & $100,0 \mathrm{a}$ & $100,0 \mathrm{a}$ \\
\hline \multicolumn{2}{|l|}{ Teste $F_{\text {trat }}$} & & $121,27 * *$ & $4550,50 * *$ & $240,51 * *$ & $227,92 * *$ & $45,11 * *$ & $75,60 * *$ \\
\hline \multicolumn{2}{|c|}{ Coeficiente de variação (\%) } & & 10,75 & 2,69 & 8,37 & 8,70 & 19,44 & 14,45 \\
\hline
\end{tabular}

${ }^{(1)}$ Médias seguidas por letras iguais, nas colunas, não diferem pelo teste de Scott-Knott, a 5\% de probabilidade. ${ }^{(2)}$ Segmentos referentes às espigas despalhadas e quebradas ao meio. ${ }^{(3)}$ Luís Eduardo Magalhães, BA; Cachoeira Dourada, MG; Sorriso, MT; Rolândia, PR; Não-Me-Toque, RS; Santa Cruz das Palmeiras, SP. ${ }^{(4)}$ Testemunha sem espigas, sem aplicação de herbicidas, plenamente capinada. ${ }^{(5)} 3 \mathrm{~T}$, soja com três trifólios verdadeiros. ${ }^{(6)} 6 \mathrm{~T}$, soja com seis trifólios verdadeiros. **Significativo a $1 \%$ de probabilidade pelo teste $\mathrm{F}$. 
et al. (2006) também recomendam aplicações de cletodim, fenoxaprope-P-etil, fluazifope-P-butil ou quizalofope-P-etil para controle de milho voluntário. Costa et al. (2014), no entanto, observaram que aplicações de cletodim foram eficientes em milho híbrido em estádio V5, porém, resultaram em níveis insatisfatórios para plantas em estádio de V8.

$\mathrm{Na}$ Tabela 4, estão apresentados os resultados do estande da cultura da soja quanto aos diferentes tratamentos, após a competição com as plantas de milho voluntário oriundas de espigas. Em quatro das seis localidades (MG, MT, RS e SP), a presença de milho voluntário afetou significativamente $\mathrm{o}$ estande da cultura da soja. Nas localidades da Bahia e do Paraná, não houve diferença de estande entre os tratamentos. Na localidade do Rio Grande do Sul, o menor estande da cultura da soja foi registrado nos tratamentos sem aplicação de cletodim (testemunha em competição). Nas demais localidades, em geral, o estande foi negativamente afetado, principalmente quando a densidade foi de 4 segmentos de espigas $\mathrm{m}^{-2}$, sobretudo na ausência da aplicação de herbicida.

$\mathrm{Na}$ Tabela 5, estão apresentados os dados de produtividade da cultura da soja obtidos para os tratamentos avaliados. Em geral, nos tratamentos em que não houve aplicação de herbicidas, observaram-se produtividades inferiores às obtidas pela testemunha capinada, com destaque para a maior densidade de segmentos de espigas, da ordem de 4 segmentos $\mathrm{m}^{-2}$, em que a produtividade foi severamente afetada. $\mathrm{Na}$ maior densidade de segmentos de espigas, observaram-se perdas de produtividade de 32,2\% na Bahia, 26,3\% em Minas Gerais, 67,0\% em Mato Grosso, 52,6\% no Paraná, 67,7\% no Rio Grande do Sul e $69,9 \%$ em São Paulo (Tabela 5). Estes resultados corroboram os de Stoller et al. (1987) e Beckett \& Stoller (1988), que comentam que quando há interferência de plantas daninhas ou voluntárias, durante todo o ciclo da soja, as perdas de rendimento são diretamente influenciadas pela densidade de infestação.

Ainda, para Beckett \& Stoller (1988), a presença de milho voluntário reduziu a produtividade da soja em 2 , $6,12,19$ e $27 \%$, quando os herbicidas foram aplicados 2, 4, 6, 8 ou 10 semanas após emergência da cultura, respectivamente. Ou seja, para a infestação oriunda de grãos, o atraso do controle prejudica o rendimento, diferentemente da infestação por plantas germinadas de espigas. Este fato é justificado, novamente, pela germinação desuniforme (fluxos) e, possivelmente mais lenta dos grãos presentes nas espigas, além da competição entre essas plantas.

Tabela 4. Estande (plantas $\mathrm{m}^{-2}$ ) da cultura da soja, após competição com milho voluntário germinado a partir segmentos de espigas, em três densidades, com ou sem aplicação do herbicida cletodim, em seis estados brasileiros ${ }^{(1)}$.

\begin{tabular}{|c|c|c|c|c|c|c|c|c|}
\hline \multirow{2}{*}{$\begin{array}{l}\text { Segmentos de } \\
\text { espigas por } \mathrm{m}^{2(2)}\end{array}$} & \multirow{2}{*}{$\begin{array}{c}\text { Cletodim } \\
\left(\mathrm{g} \mathrm{ha}^{-1}\right)\end{array}$} & \multirow{2}{*}{$\begin{array}{l}\text { Momento de } \\
\text { aplicação }\end{array}$} & \multicolumn{6}{|c|}{ Local de desenvolvimento do experimento $^{(3)}$} \\
\hline & & & BA & MG & MT & PR & RS & SP \\
\hline \multicolumn{3}{|c|}{ Testemunha absoluta capinada ${ }^{(4)}$} & 35,8 & $31,3 b$ & $39,3 \mathrm{a}$ & 46,5 & $51,0 \mathrm{a}$ & $40,8 \mathrm{a}$ \\
\hline 1 & Ausente & - & 40,8 & $31,5 \mathrm{~b}$ & $44,5 \mathrm{a}$ & 48,5 & $40,8 b$ & $37,3 \mathrm{a}$ \\
\hline 2 & Ausente & - & 37,3 & $25,5 b$ & $38,5 \mathrm{a}$ & 47,0 & $43,3 b$ & $35,3 \mathrm{a}$ \\
\hline 4 & Ausente & - & 41,0 & $35,5 \mathrm{a}$ & $25,3 \mathrm{c}$ & 43,5 & $38,8 \mathrm{~b}$ & $18,8 \mathrm{~b}$ \\
\hline 1 & 108 & $3 \mathrm{~T}^{(5)}$ & 37,5 & $32,0 \mathrm{~b}$ & $43,5 \mathrm{a}$ & 47,3 & $54,5 \mathrm{a}$ & $44,0 \mathrm{a}$ \\
\hline 2 & 108 & $3 \mathrm{~T}$ & 39,3 & $35,0 \mathrm{a}$ & $40,0 \mathrm{a}$ & 48,5 & $51,5 \mathrm{a}$ & $38,8 \mathrm{a}$ \\
\hline 4 & 108 & $3 \mathrm{~T}$ & 40,0 & $27,8 b$ & $40,3 \mathrm{a}$ & 46,0 & $49,8 \mathrm{a}$ & $37,0 \mathrm{a}$ \\
\hline 1 & 108 & $6 \mathrm{~T}^{(6)}$ & 43,3 & $29,8 b$ & $41,5 \mathrm{a}$ & 50,8 & $50,5 \mathrm{a}$ & $42,0 \mathrm{a}$ \\
\hline 2 & 108 & $6 \mathrm{~T}$ & 39,0 & $31,8 \mathrm{~b}$ & $44,0 \mathrm{a}$ & 51,0 & $50,3 \mathrm{a}$ & $36,0 \mathrm{a}$ \\
\hline 4 & 108 & $6 \mathrm{~T}$ & 44,3 & $29,5 b$ & $31,8 b$ & 51,0 & $47,5 \mathrm{a}$ & $38,3 \mathrm{a}$ \\
\hline 1 & 108 e 84 & $3 \mathrm{~T}$ e $6 \mathrm{~T}$ & 42,3 & $36,5 \mathrm{a}$ & $42,0 \mathrm{a}$ & 49,3 & $51,0 \mathrm{a}$ & $37,5 \mathrm{a}$ \\
\hline 2 & 108 e 84 & $3 \mathrm{~T}$ e $6 \mathrm{~T}$ & 45,3 & $38,0 \mathrm{a}$ & $44,0 \mathrm{a}$ & 46,3 & $50,0 \mathrm{a}$ & $40,5 \mathrm{a}$ \\
\hline 4 & 108 e 84 & $3 \mathrm{~T}$ e $6 \mathrm{~T}$ & 37,5 & $36,3 \mathrm{a}$ & $41,0 \mathrm{a}$ & 47,0 & $49,3 \mathrm{a}$ & $39,8 \mathrm{a}$ \\
\hline \multicolumn{3}{|l|}{ Teste $\mathrm{F}_{\text {trat }}$} & $0,93^{\mathrm{ns}}$ & $2,40^{*}$ & $6,32 * *$ & $1,34^{\mathrm{ns}}$ & $3,02 * *$ & $3,86^{* *}$ \\
\hline \multicolumn{3}{|c|}{ Coeficiente de variação (\%) } & 15,02 & 14,88 & 10,97 & 8,05 & 10,93 & 16,64 \\
\hline
\end{tabular}

${ }^{(1)}$ Médias seguidas por letras iguais, nas colunas, não diferem pelo teste de Scott-Knott, a $5 \%$ de probabilidade. ${ }^{(2)}$ Segmentos referentes às espigas despalhadas e quebradas ao meio. ${ }^{(3)}$ Luís Eduardo Magalhães, BA; Cachoeira Dourada, MG; Sorriso, MT; Rolândia, PR; Não-Me-Toque, RS; Santa Cruz das Palmeiras, SP. ${ }^{(4)}$ Testemunha sem espigas, sem aplicação de herbicidas, plenamente capinada. ${ }^{(5)} 3 \mathrm{~T}$, soja com três trifólios verdadeiros. ${ }^{(6)} 6 \mathrm{~T}$, soja com seis

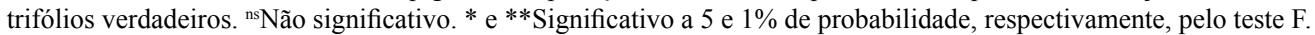


Tabela 5. Produtividade $\left(\mathrm{kg} \mathrm{ha}^{-1}\right)$ da cultura da soja após competição com milho voluntário, germinado a partir de segmentos de espigas, em três diferentes densidades, com ou sem aplicação do herbicida cletodim, em seis estados brasileiros ${ }^{(1)}$.

\begin{tabular}{|c|c|c|c|c|c|c|c|c|}
\hline \multirow{2}{*}{$\begin{array}{l}\text { Segmentos de } \\
\text { espigas por } \mathrm{m}^{2(2)}\end{array}$} & \multirow{2}{*}{$\begin{array}{l}\text { Cletodim } \\
\left(\mathrm{g} \mathrm{ha}^{-1}\right)\end{array}$} & \multirow{2}{*}{$\begin{array}{l}\text { Momento de } \\
\text { aplicação }\end{array}$} & \multicolumn{6}{|c|}{ Local de desenvolvimento do experimento ${ }^{(3)}$} \\
\hline & & & $\mathrm{BA}$ & MG & MT & PR & RS & SP \\
\hline \multicolumn{3}{|c|}{ Testemunha absoluta capinada $^{(4)}$} & $3691,4 \mathrm{a}$ & $3195,7 \mathrm{a}$ & $3100,6 a$ & $4397,5 \mathrm{a}$ & $3820,9 a$ & $4721,8 \mathrm{a}$ \\
\hline 1 & Ausente & - & $3399,5 \mathrm{a}$ & $2617,8 b$ & $2566,5 \mathrm{a}$ & $3476,3 b$ & $3073,3 \mathrm{c}$ & $3734,5 b$ \\
\hline 2 & Ausente & - & $3037,3 b$ & $1751,2 \mathrm{~b}$ & $2744,6 \mathrm{a}$ & $3051,5 b$ & $2349,4 d$ & $2754,9 \mathrm{c}$ \\
\hline 4 & Ausente & - & $2501,4 \mathrm{c}$ & $2355,4 \mathrm{~b}$ & $1022,2 b$ & $2081,1 \mathrm{c}$ & $1234,6 \mathrm{e}$ & $1284,3 \mathrm{~d}$ \\
\hline 1 & 108 & $3 \mathrm{~T}^{(5)}$ & $3350,0 \mathrm{a}$ & $2597,3 b$ & $2469,1 \mathrm{a}$ & $4434,8 \mathrm{a}$ & $3583,8 b$ & $4672,6 a$ \\
\hline 2 & 108 & $3 \mathrm{~T}$ & $3541,6 \mathrm{a}$ & $2787,7 \mathrm{~b}$ & $1894,0 \mathrm{~b}$ & $3888,4 \mathrm{a}$ & $3701,4 b$ & $4494,7 \mathrm{a}$ \\
\hline 4 & 108 & $3 \mathrm{~T}$ & $3446,6 a$ & $2185,2 b$ & $1809,9 b$ & $3868,2 \mathrm{a}$ & $3584,8 b$ & $3869,1 \mathrm{~b}$ \\
\hline 1 & 108 & $6 \mathrm{~T}^{(6)}$ & $3786,7 \mathrm{a}$ & $3327,8 \mathrm{a}$ & $3197,8 \mathrm{a}$ & $4605,5 \mathrm{a}$ & $3810,6 a$ & $4730,4 a$ \\
\hline 2 & 108 & $6 \mathrm{~T}$ & $3927,2 \mathrm{a}$ & $3311,3 \mathrm{a}$ & $3285,5 \mathrm{a}$ & $4499,0 \mathrm{a}$ & $3920,0 \mathrm{a}$ & $4132,3 \mathrm{~b}$ \\
\hline 4 & 108 & $6 \mathrm{~T}$ & $3642,0 \mathrm{a}$ & $3175,6 \mathrm{a}$ & $2592,5 \mathrm{a}$ & $3935,6 a$ & $3602,5 b$ & $4238,8 b$ \\
\hline 1 & 108 e 84 & $3 \mathrm{~T}$ e $6 \mathrm{~T}$ & $3731,6 a$ & $3390,0 \mathrm{a}$ & $3177,9 \mathrm{a}$ & $4176,6 a$ & $3946,3 a$ & $4825,6 \mathrm{a}$ \\
\hline 2 & 108 e 84 & $3 \mathrm{~T}$ e $6 \mathrm{~T}$ & $3761,5 \mathrm{a}$ & $3244,8 \mathrm{a}$ & $3372,8 \mathrm{a}$ & $4484,9 \mathrm{a}$ & $4040,5 \mathrm{a}$ & $4953,1 \mathrm{a}$ \\
\hline 4 & 108 e 84 & $3 \mathrm{~T}$ e $6 \mathrm{~T}$ & $3872,3 \mathrm{a}$ & $3358,6 \mathrm{a}$ & $2938,2 \mathrm{a}$ & $4325,0 \mathrm{a}$ & $4076,9 \mathrm{a}$ & $4795,6 \mathrm{a}$ \\
\hline \multicolumn{3}{|l|}{ Teste $\mathrm{F}_{\text {trat }}$} & $7,01 * *$ & $3,98 * *$ & $3,05 * *$ & $8,60 * *$ & $26,28 * *$ & $18,72 * *$ \\
\hline \multicolumn{3}{|c|}{ Coeficiente de variação (\%) } & 8,29 & 18,56 & 30,22 & 12,42 & 9,17 & 11,73 \\
\hline
\end{tabular}

${ }^{(1)}$ Médias seguidas por letras iguais, nas colunas, não diferem pelo teste de Scott-Knott, a $5 \%$ de probabilidade. (2)Segmentos referentes às espigas despalhadas e quebradas ao meio. ${ }^{(3)}$ Luís Eduardo Magalhães, BA; Cachoeira Dourada, MG; Sorriso, MT; Rolândia, PR; Não-Me-Toque, RS; Santa Cruz das Palmeiras, SP. ${ }^{(4)}$ Testemunha sem espigas, sem aplicação de herbicidas, plenamente capinada. ${ }^{(5)} 3 \mathrm{~T}$, soja com três trifólios verdadeiros. ${ }^{(6)} 6 \mathrm{~T}$, soja com seis trifólios verdadeiros. ${ }^{*}$ Significativo a $1 \%$ de probabilidade pelo teste $\mathrm{F}$.

Quando não é controlado na cultura da soja, o milho voluntário compete por recursos de forma similar à das plantas daninhas. Marquardt et al. (2012) observaram perdas de até $41 \%$ da produtividade de soja, quando ocorreu emergência precoce do milho voluntário, com destaque para a densidade de 16 plantas $\mathrm{m}^{-2}$. Quando o milho teve emergência tardia (semeado 21 dias após a soja), não se registrou perda de rendimento da soja. Assim, Marquardt et al. (2012) atribuem parte da interferência negativa do milho voluntário na cultura da soja à competição por luz, tal como outras plantas daninhas.

A produtividade obtida nos tratamentos com uso de herbicidas está coerente com os dados de controle (Tabelas 2 e 3), ou seja, quando em baixa densidade de infestação de milho voluntário, uma aplicação de cletodim em estádio de 6T assegurou a produtividade. Em alta densidade de infestação (4 segmentos de espigas $\mathrm{m}^{-2}$ ), a produtividade da soja foi assegurada em quatro das seis localidades experimentais, com uma aplicação de cletodim em estádio de $6 \mathrm{~T}$ e, em todas as seis localidades, com duas aplicações do graminicida. Marquardt \& Johnson (2013) também obtiveram produtividade plena da cultura da soja, por meio de pulverizações com cletodim (79 $\left.\mathrm{g} \mathrm{ha}^{-1}\right)$, porém em associação com glifosato $\left(840 \mathrm{~g} \mathrm{ha}^{-1}\right)$.

\section{Conclusões}

1. A presença de plantas voluntárias de milho, originadas de segmentos de espigas, reduz em até $69,9 \%$ a produtividade da cultura da soja cultivada em sucessão.

2. A produtividade da soja é assegurada por meio do controle eficaz das plantas voluntárias de milho tolerante ao glifosato oriundas de espigas, com aplicação única de cletodim $\left(108 \mathrm{~g} \mathrm{ha}^{-1}\right)$ no estádio de seis trifólios da soja; ou por meio de duas aplicações sequenciais do graminicida com a soja com três e seis trifólios.

\section{Referências}

ANDERSEN, R.N. Control of volunteer corn and giant foxtail in soybeans. Weed Science, v.24, p.253-256, 1976.

ARTUZI, J.P.; CONTIERO, R.L. Herbicidas aplicados na soja e produtividade do milho em sucessão. Pesquisa Agropecuária Brasileira, v.41, p.1119-1123, 2006.

BECKETT, T.H.; STOLLER, E.W. Volunteer corn (Zea mays) interference in soybeans (Glycine max). Weed Science, v.36, p.159-166, 1988.

BRAGACHINI, M.; PEIRETTI, J. Maíz en Argentina: un cultivo clave para una agricultura sustentable. Disponível em: $<$ http://www.agriculturadeprecision.org/articulos/cosecha/ 
Maiz-En-Argentina-Cultivo-Clave-Para-Agricultura-Sustentable. asp>. Acesso em: 6 set. 2005.

CLEWIS, S.B.; THOMAS, W.E.; EVERMAN, W.J.; WILCUT, J.W. Glufosinate-resistant corn interference in glufosinate-resistant cotton. Weed Technology, v.22, p.211-216, 2008.

COSTA, N.V.; ZOBIOLE, L.H.S.; SCARIOT, C.A.; PEREIRA, G.R.; MORATELLI, G. Glyphosate tolerant volunteer corn control at two development stages. Planta Daninha, v.32, p.675-682, 2014. DOI: $10.1590 / \mathrm{S} 0100-83582014000400002$.

DEEN, W.; HAMILL, A.; SHROPSHIRE, C.; SOLTANI, N.; SIKKEMA, P.H. Control of volunteer glyphosate-resistant corn (Zea mays) in glyphosate-resistant soybean (Glycine max). Weed Technology, v.20, p.261-266, 2006. DOI: 10.1614/WT-02-128.1.

MACIEL, C.D. de G.; ZOBIOLE, L.H.S.; SOUZA, J.I. de; HIROOKA, E.; LIMA, L.G.N.V. de; SOARES, C.R.B.; PIVATTO, R.A.D.; FUCHS, G.M.; HELVIG, E.O. Eficácia do herbicida haloxyfop R (GR-142) isolado e associado ao 2,4-D no controle de híbridos de milho $\mathrm{RR}^{\circledR}$ voluntário. Revista Brasileira de Herbicidas, v.12, p.112-123, 2013. DOI: 10.7824/rbh.v12i2.244.

MARCA, V.; PROCÓPIO, S. de O.; SILVA, A.G. da; VOLF, M. Chemical control of glyphosate-resistant volunteer maize. Revista Brasileira de Herbicidas, v.14, p.103-110, 2015. DOI: 10.7824/ rbh.v14i2.408.

MARQUARDT, P.; JOHNSON, W.G. Influence of clethodim application timing on control of volunteer corn in soybean. Weed Technology, v.27, p.645-648, 2013. DOI: 10.1614/ WT-D-12-00188.1.

MARQUARDT, P.; KRUPKE, C.; JOHNSON, W.G. Competition of transgenic volunteer corn with soybean and the effect on western corn rootworm emergence. Weed Science, v.60, p.193-198, 2012. DOI: 10.1614/WS-D-11-00133.1.

PEARSON, E.S.; HARTLEY, H.O. (Ed.). Biometrika tables for statisticians. Cambridge: University Press, 1956. 238p.

PETTER, F.A.; SIMA, V.M.; FRAPORTI, M.B.; PEREIRA, C.S.; PROCÓPIO, S.O.; SILVA, A.F. Volunteer RR ${ }^{\circledR}$ corn management in roundup ready ${ }^{\circledR}$ soybean-corn succession system. Planta Daninha, v.33, p.119-128, 2015. DOI: 10.1590/S0100-83582015000100014.
PUZZI, D. Abastecimento e armazenagem de grãos. Campinas: Instituto Campineiro de Ensino Agrícola, 1986. 603p.

RODRIGUES, B.N.; ALMEIDA, F.S. de. Guia de herbicidas. 6.ed. Londrina: Ed. dos Autores, 2011. 697p.

SCOTT, A.J.; KNOTT, M.A cluster analysis method for grouping means in the analysis of variance. Biometrics, v. 30, p.507-512, 1974. DOI: $10.2307 / 2529204$.

SOLTANI, N.; SHROPSHIRE, C.; SIKKEMA, P.H. Control of volunteer glyphosate-tolerant maize (Zea mays) in glyphosate-tolerant soybean (Glycine max). Crop Protection, v.25, p.178-181, 2006. DOI: 10.1016/j.cropro.2005.03.017.

SOUZA, C.M.A. de; RAFULL, L.Z.L.; REIS, E.F. dos; ALVES SOBRINHO, T. Perdas na colheita mecanizada de milho em agricultura familiar da Zona da Mata mineira. Revista Brasileira de Milho e Sorgo, v.5, p.280-290, 2006. DOI: 10.18512/1980-6477/ rbms.v5n2p280-290.

STECKEL, L.E.; THOMPSON, M.A.; HAYES, R.M. Herbicide options for controlling glyphosate-tolerant corn in a corn replant situation. Weed Technology, v.23, p.243-246, 2009. DOI: 10.1614/ WT-08-151.1.

STOLLER, E.W.; HARRISON, S.K.; WAX, L.M.; REGNIER, E.E.; NAFZIGER, E.D. Weed interference in soybeans (Glycine max). In: FOY, C.L. Reviews of weed science. Champaign: Weed Science Society of America, 1987. v.3, p.155-181.

THOMAS, W.E.; EVERMAN, W.J.; CLEWIS, S.B.; WILCUT, J.W. Glyphosate-resistant corn interference in glyphosate-resistant cotton. Weed Technology, v.21, p.372-377, 2007. DOI: 10.1614/ WT-06-007.1.

YORK, A.C.; STEWART, A.M.; VIDRINE, P.R.; CULPEPPER, A.S. Control of volunteer glyphosate-resistant cotton in glyphosate-resistant soybean. Weed Technology, v.18, p.532-539, 2004. DOI: 10.1614/WT-03-073R1.

YOUNG, B.G.; HART, S.E. Control of volunteer sethoxydim-resistant corn (Zea mays) in soybean (Glycine max). Weed Technology, v.11, p.649-655, 1997.

Recebido em 8 de maio de 2015 e aprovado em 24 de fevereiro de 2016 